\title{
Self-Similarity Techniques for Chaotic Attractors with Many Scrolls Using Step Series Switching
}

\author{
Emile Franc Doungmo Goufo ${ }^{a}$, \\ Chokkalingam Ravichandran $^{b}$ and Gunvant A. Birajdar ${ }^{c}$ \\ ${ }^{a}$ Department of Mathematical Sciences, University of South Africa \\ 0003 Florida, South Africa \\ ${ }^{b}$ Department of Mathematics, Kongunadu Arts and Science college(Autonomous) \\ 641029 Coimbatore, India \\ ${ }^{c}$ School of Rural Development, Tata Institute of Social Sciences. \\ 413601 Tuljapur, India \\ E-mail(corresp.): franckemile2006@yahoo.ca
}

Received October 6, 2020; revised September 24, 2021; accepted September 24, 2021

\begin{abstract}
Highly applied in machining, image compressing, network traffic prediction, biological dynamics, nerve dendrite pattern and so on, self-similarity dynamic represents a part of fractal processes where an object is reproduced exactly or approximately exact to a part of itself. These reproduction processes are also very important and captivating in chaos theory. They occur naturally in our environment in the form of growth spirals, romanesco broccoli, trees and so on. Seeking alternative ways to reproduce self-similarity dynamics has called the attention of many authors working in chaos theory since the range of applications is quite wide. In this paper, three combined notions, namely the step series switching process, the Julia's technique and the fractal-fractional dynamic are used to create various forms of self-similarity dynamics in chaotic systems of attractors, initially with two, five and seven scrolls. In each case, the solvability of the model is addressed via numerical techniques and related graphical simulations are provided. It appears that the initial systems are able to trigger a self-similarity process that generates the exact or approximately exact copy of itself or part of itself. Moreover, the dynamics of the copies are impacted by some model's parameters involved in the process. Using mathematical concepts to re-create features that usually occur in a natural way proves to be a prowess as related applications are many for engineers.
\end{abstract}

Copyright (C) 2021 The Author(s). Published by Vilnius Gediminas Technical University This is an Open Access article distributed under the terms of the Creative Commons Attribution License (http://creativecommons.org/licenses/by/4.0/), which permits unrestricted use, distribution, and reproduction in any medium, provided the original author and source are credited. 
Keywords: mathematical and engineering model, switching process, self-organization, fractal and fractional process, numerical method.

AMS Subject Classification: 28A80; 37F50; 26A33; 33F05.

\section{Introduction}

Chaotic bifurcations, fractal processes, self-organization, auto-duplication, selfassembly all started with the concept of dynamical system. The theory of dynamical system has been studied for more than a century as it has never stopped wondering scientists with its unpredictable dynamical systems which cover a large number of applications related to scientific domains such as (applied) mathematics, engineering, physics, biology, electronics, economics and even medical sciences $[6,11,13,16,18]$. One of the major applications of the theory of dynamical systems is the study of chaotic systems that represents the fundamentals of chaos theory and also of bifurcation theory. There are many types of dynamical systems $[1,7,10,11,12,16,17,19]$, including the classical dynamical system depicted in Figure 1 and the open dynamical system depicted in Figure 2 where we can observe different orbits or trajectories.

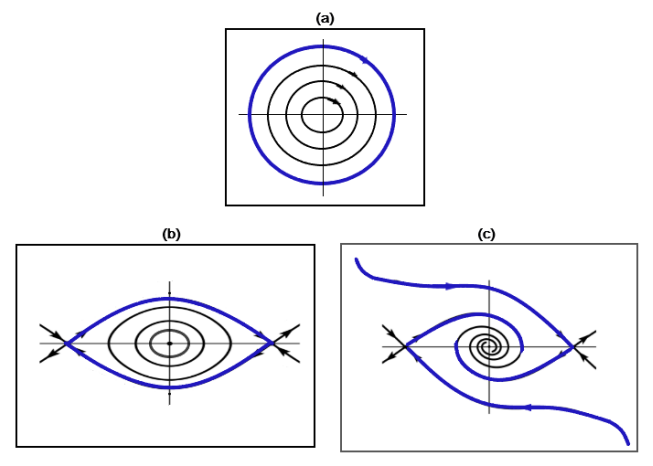

Figure 1. Illustration of the basic principles of open dynamical systems. In (a), we obverse a basic process depicting the phase portrait for a harmonic oscillator happening in a plane and where the only fixed point is the origin and the circles represent the other trajectories. In (b), we obverse another basic process depicting the phase portrait of undamped pendulum happening in a cylinder and where trajectories are closed curves with some going around the origin and other wrapping around the cylinder. Lastly, in (c), we obverse a process depicting phase portrait for a damped pendulum with the difference that the origin has become an attracting fixed point.

In Figure 1 (a), we obverse a basic process depicting the phase portrait for a harmonic oscillator happening in a plane and where the only fixed point is the origin and the circles represent the other trajectories. In Figure 1 (b), we obverse another basic process depicting the phase portrait of undamped pendulum happening in a cylinder and where trajectories are closed curves with some going around the origin and other wrapping around the cylinder. Lastly, in Figure 1 (c), we obverse a process depicting phase portrait for a damped 
pendulum with the difference that the origin has become an attracting fixed point.

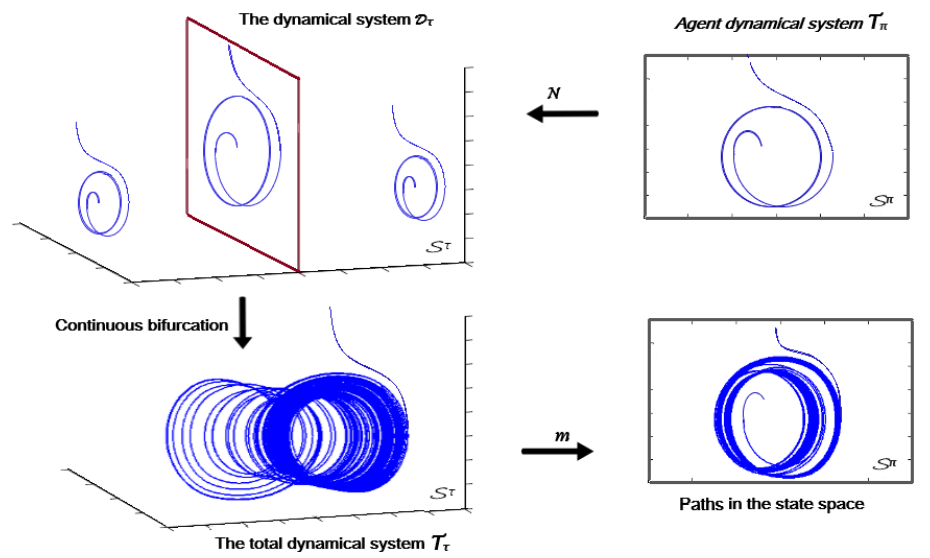

Figure 2. Basic principle of open dynamical system. We can see the initial behavior in isolation of an agent dynamical system $\mathcal{T}_{\pi}$ correctly defined on its suitable space $\mathcal{S}_{\pi}$ (upper right)). The process continues at the upper left-side where we can see how the decoupled agent dynamical system $\mathcal{D}_{\pi}$, defined on its suitable total space $\mathfrak{S}_{\tau}$ joint together to form the total system $\mathcal{T}_{\tau}$ depicted in the lower left-side. It ends in the lower right-side which depicts the projection or paths showing how an agent behaves in a particular environment $\left(\mathcal{S}_{\pi}\right)$. These last paths in the state $\mathcal{S}_{\pi}$ are the ones which make the major contrast here and that particularly characterize the so-called open system. Note the difference between the orbits for the agent in isolation (upper right) and those in the open phase portrait for the embedded agent dynamical system (lower right)), which are shown to overlap.

In Figure 2 we have the illustration of an open dynamical system where we can see the initial behavior in isolation of an agent dynamical system $\mathcal{T}_{\pi}$ correctly defined on its suitable space $\mathcal{S}_{\pi}$ (Figure 2 (upper right)). The process continues with Figure 2 (upper left) where we can see how the decoupled agent dynamical system $\mathcal{D}_{\pi}$, defined on its suitable total space $\mathfrak{S}_{\tau}$ joint together to form the total system $\mathcal{T}_{\tau}$ depicted in Figure 2 (lower left). It ends in Figure 2 (lower right) which depicts the projection or paths showing how an agent behaves in a particular environment $\left(\mathcal{S}_{\pi}\right)$. These last paths in the state $\mathcal{S}_{\pi}$ are the ones which make the major contrast here and that particularly characterize the so-called open system. Indeed, we can observe difference between the orbits for the agent in isolation (Figure 2 (upper right)) and those in the open phase portrait for the embedded agent dynamical system (Figure 2 (lower right)), which are shown to overlap. Hence, it is then more difficult to analyze trajectories in open dynamical systems.

Fractal processes are also depicted in dynamical systems with a simple principle as shown in Figure 3 where we can see an example of auto-replication structures coupled with self-similar fluctuations. Indeed the tree-like structures (Figure 2 (bottom)), identified here as spatial fractal comprise branches disposed in a self-similar process. There is an obvious resemblance between smallscale tree-like structure and bigger ones. Such resemblance process couples 

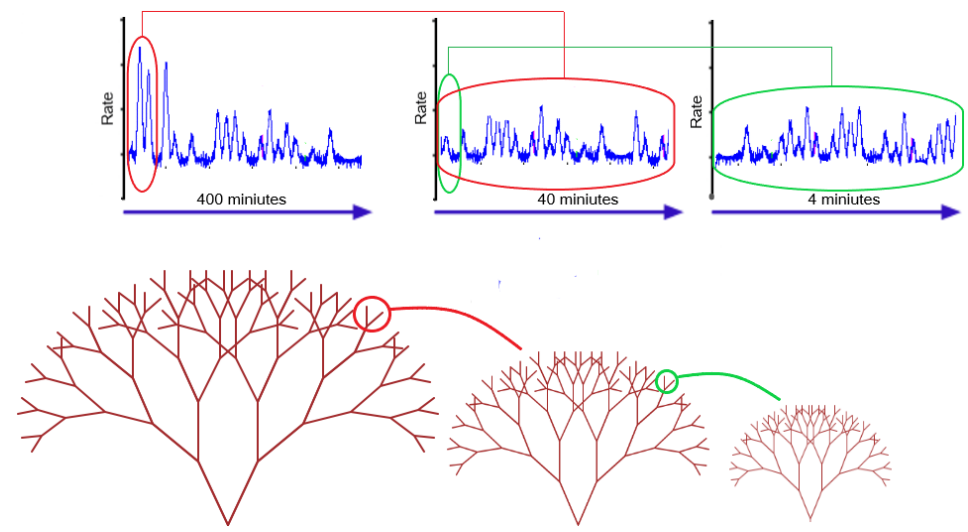

Figure 3. Basic principle of auto-replication (self-similar) dynamics. The tree-like structures (bottom), identified here as spatial fractal comprise branches disposed in a self-similar process that couples with a fractal temporal process, the regulation rates (top), which generate different fluctuations based on variable time scales. The said fluctuations are also shown be statistically self-similar.

with a fractal temporal process, like for instance the regulation rates (Figure 2 (top)), which generate different variations (or fluctuations) based on variable time scales. The said fluctuations are also shown be statistically self-similar. In the next sections, we are going to use different processes to reproduce selfsimilarity in dynamical systems.

\section{Self-similarity in classical chaotic attractors with many scrolls using step series switching}

In the work [15] the authors introduced a method of step series switching able to generate chaotic attractors with many scrolls from some simple linear models. The following piecewise-linear function switching system was then proposed:

$$
\frac{d X}{d t}=M_{1}\left[X-s\left({ }^{T} M_{2} X-4\right) M_{3}\right]
$$

with

$$
\begin{aligned}
& X=X(t)=(x(t), y(t), z(t))^{T}, \quad s(\zeta)= \begin{cases}1, & \text { when } \zeta>0, \\
0, & \text { when } \zeta \leq 0\end{cases} \\
& M_{1}=\left(\begin{array}{ccc}
\frac{1}{2} & 10 & 0 \\
-10 & \frac{1}{2} & 0 \\
0 & 0 & -10
\end{array}\right), M_{2}={ }^{T}(d, 4,4), \quad M_{3}={ }^{T}(0,1,1),
\end{aligned}
$$

where $d$ is a real number which, when vanishes $(d=0)$ causes the model $(2.1)$ to generate a chaotic attractor with two scrolls, as depicted in Figure 4. 


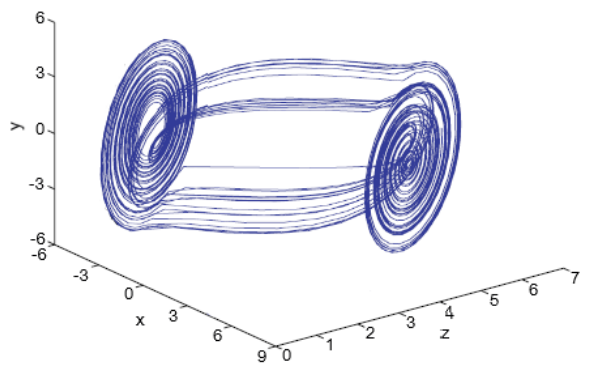

Figure 4. A chaotic attractor with two scrolls. The double-scroll attractor is generated by (2.1) via the series switching technique with the parameter value $d=0$.

The modification of the above function $s(\zeta)$ into

$$
s(\zeta)= \begin{cases}s_{1}, & \text { when } \zeta \leq b_{1}, \\ s_{2}, & \text { when } b_{i-1}<\zeta \leq b_{i} \text { for } i=2,3, \ldots, n-1, \\ s_{n}, & \text { when } \zeta>b_{n},\end{cases}
$$

with $\left\{s_{i}\right\}_{1 \leq i \leq n}$ and $\left\{b_{i}\right\}_{1 \leq i \leq n}$ representing series of constants that is strictly increasing. The model (2.1) therefore takes the form

$$
\frac{d X}{d t}=\tilde{M}_{1}\left[X-s\left({ }^{T} \tilde{M}_{2} X-4\right) \tilde{M}_{3}\right]
$$

with

$$
\tilde{M}_{1}=\left(\begin{array}{ccc}
a & c & 0 \\
-c & a & 0 \\
0 & 0 & -e
\end{array}\right), \quad \tilde{M}_{2}=^{T}(0, f, f) \text { and } \tilde{M}_{3}={ }^{T}(0,1,1) .
$$

For the parameter values

$$
\begin{aligned}
& \left(b_{1}, b_{2}, b_{3}, b_{4}\right)=\left(-\frac{1}{2}, \frac{1}{2}, \frac{3}{2}, \frac{5}{2}\right),\left(s_{1}, s_{2}, s_{3}, s_{4}, s_{5}\right)=(-1,0,1,2,3), \\
& (a, c, e, f)=(1 / 2,10,5,1 / 2),
\end{aligned}
$$

the model (2.2) generates a chaotic attractor with five scrolls, as depicted in Figure 5 .

There also exists circuit diagram, able to produce a similar result, like the one depicted in Figure 6 that was proposed in [14] and whose equation reads as

$$
\left(\begin{array}{c}
\frac{d u_{1}}{d t} \\
\frac{d u_{2}}{d t} \\
\frac{d u_{3}}{d t}
\end{array}\right)=\frac{1}{C}\left(\begin{array}{ccc}
0 & \frac{1}{R_{f 21}} & 0 \\
\frac{-1}{R_{f 12}} & \frac{1}{R_{f 22}} & 0 \\
0 & 0 & \frac{-1}{R_{f 33}}
\end{array}\right)\left(\begin{array}{c}
u_{1}-W\left(u_{1}+u_{3}\right) \\
u_{2}-W\left(u_{2}+u_{3}\right) \\
u_{3}-\frac{1}{2} W\left(u_{1}+u_{3}\right)-\frac{1}{2} W\left(u_{2}+u_{3}\right)
\end{array}\right),
$$

where $W(\eta)= \begin{cases}0, & \text { when } \eta<\frac{V_{C C}}{m}, \\ i \frac{V_{C C}}{m}, & \text { when }(2 i-1) \frac{V_{C C}}{m} \leq \eta<(2 i+1) \frac{V_{C C}}{m} \text { for } i=2,3, \ldots, n-1, \\ n \frac{V_{C C}}{m}, & \text { when } \eta \geq(2 n-1) \frac{V_{C C}}{m}\end{cases}$ 


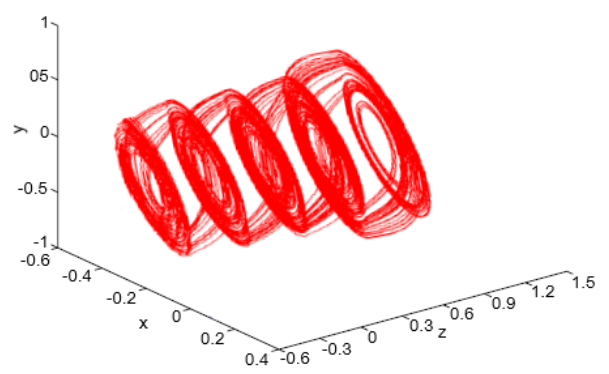

Figure 5. A chaotic attractor with five scrolls. The quintuple-scroll attractor is generated by $(2.2)$ via the series switching technique with the parameter values $\left(b_{1}, b_{2}, b_{3}, b_{4}\right)=\left(-\frac{1}{2}, \frac{1}{2}, \frac{3}{2}, \frac{5}{2}\right),\left(s_{1}, s_{2}, s_{3}, s_{4}, s_{5}\right)=(-1,0,1,2,3) ;(a, c, e, f)=\left(\frac{1}{2}, 10,5, \frac{1}{2}\right)$.

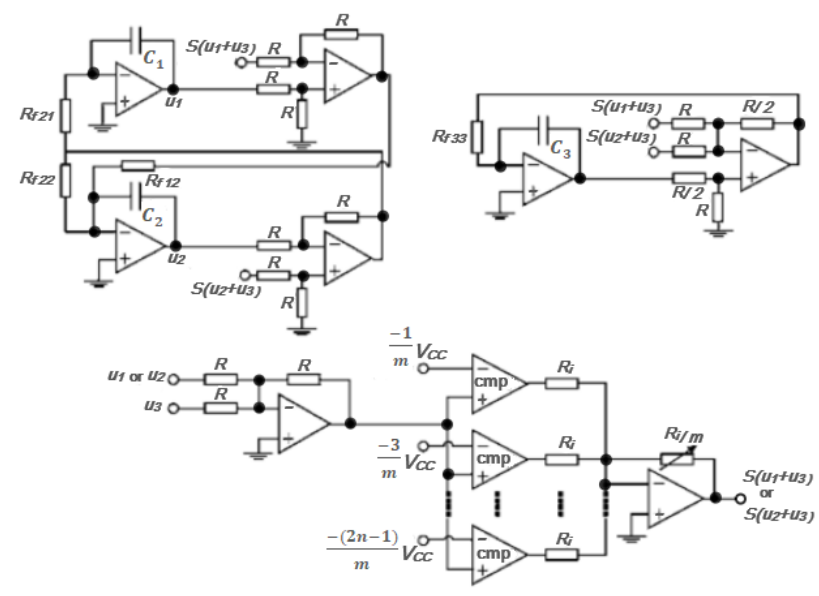

Figure 6. Circuit diagram, able to produce a chaotic attractor with seven scrolls.

The system (2.3) is transformed into the dimensionless state model that reads as

$$
\left\{\begin{array}{l}
\frac{d x}{d \tau}=y-W(y+z) \\
\frac{d y}{d \tau}=-x+b y+W(x+z)-b W(y+z) \\
\frac{d z}{d \tau}=-z+d W(x+z)-d W(y+z)
\end{array}\right.
$$

with $W(\eta)=0.5 \sum \frac{i=0}{n} v(\eta-i-0.5)$, where $v(\cdot)$ represents the unit step function and where we have set

$$
\tau=t / R C, R=3 k \Omega, \quad C=100 p F, \quad x=u_{1} / 1 V, \quad y=u_{2} / 1 V, \quad z=u_{3} / 1 V .
$$

Henceforth, the model (2.4) generates a chaotic attractor with seven scrolls, as depicted in Figure 7 for the parameter values

$$
(b, d, W(\eta))=\left(\frac{1}{10}, \frac{1}{2}, \frac{1}{2} v\left(\eta-\frac{1}{2}\right)+\frac{1}{2} v\left(\eta-\frac{3}{2}\right)\right) .
$$




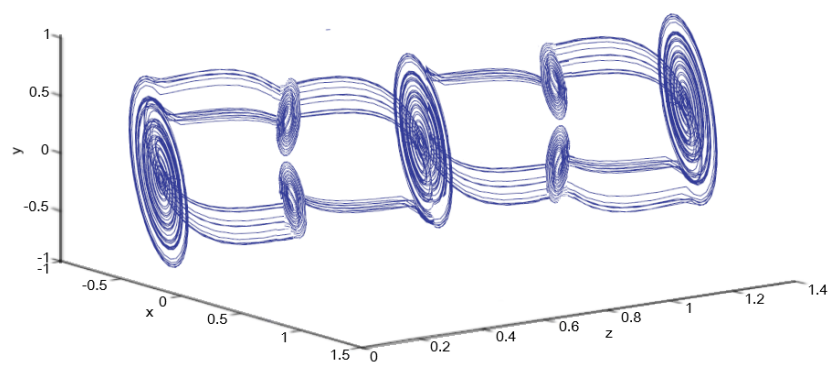

Figure 7. A chaotic attractor with seven scrolls. The 7 -scroll attractor is generated by (2.4) via the series switching technique with the parameter values $(b, d, W(\eta))=\left(\frac{1}{10}, \frac{1}{2}, \quad \frac{1}{2} v\left(\eta-\frac{1}{2}\right)+\frac{1}{2} v\left(\eta-\frac{3}{2}\right)\right)$.

\section{$3 \quad$ Fractal (Julia's) process in self-similarity for attractors with many scrolls}

As proposed in the article [4], we consider a vector state $U_{j}=\left(g_{j}, h_{j}\right)$. Then, Julia's process is a technique that consists of getting the subsequent term $U_{j+1}$. Take the complex number $\mathbb{Z}_{j}=g_{j}+i h_{j}$ hence we have $\mathbb{Z}_{j+1}=\mathbb{Z}_{j}^{2}$, that is equivalent to

$$
\left\{\begin{array}{l}
g_{j+1}=g_{j}^{2}-h_{j}^{2}, \\
h_{j+1}=2 g_{j} h_{j} .
\end{array}\right.
$$

The inverse map is established by expressing $g_{j}$ and $h_{j}$ in terms of $g_{j+1}$ and $h_{j+1}$. Therefore, we obtain the dynamics that takes the form $U_{j+1}=$ $\left(g_{j+1}, h_{j+1}\right)=B\left(g_{j}, h_{j}\right)$ with $B$ denoting the treatment process. Hence, the real part and imaginary part are separately computed following the Algorithm 1 .

More generally, fractal processes consider the complete metric unit denoted here by $\mathbf{F}$. We can therefore consider fractal processes system, namely $\Psi$ and defined in $\mathbf{F}$ by

$$
\Psi: \mathbf{F} \rightarrow \mathbf{F} \text { so that } \Psi\left(g_{j}, h_{j}\right)=\left(g_{m}, h_{m}\right) .
$$

Hence, the system $\Psi$ takes the iterative representation:

$$
\Psi \begin{cases}\left(g_{0}, h_{0}\right), & \\ \left(g_{j+1}, h_{j+1}\right)= & B_{1}\left(\theta g_{j}+\gamma, \beta h_{j}+\lambda\right), \\ \left(g_{j+2}, h_{j+2}\right)= & B_{2}\left(g_{j+1}, h_{j+1}\right), \\ \left(g_{j+3}, h_{j+3}\right)= & K_{1}\left(g_{j+2}, h_{j+2}\right), \\ \left(g_{j+4}, h_{j+4}\right)= & B_{3}\left(g_{j+3}, h_{j+3}\right), \\ \vdots & \\ \left(g_{k+1}, h_{k+1}\right)= & K_{l}\left(g_{k-1}, h_{k-1}\right), \\ \vdots & \\ \left(g_{m}, h_{m}\right)= & B_{m-l}\left(g_{m-1}, h_{m-1}\right),\end{cases}
$$


Algorithm 1. Computing $U_{j+1}=\left(g_{j+1}, h_{j+1}\right)=B\left(g_{j}, h_{j}\right)$

1: if $g_{j}<0$ then

2: $\quad g_{j+1}=\left(\frac{g_{j}}{2}+\left[g_{j}^{2}+h_{j}^{2}\right]^{\frac{1}{2}}\right)^{\frac{1}{2}}$

3: $\quad h_{j+1}=\frac{h_{j}}{2 h_{j+1}}$

4: end if

5: if $g_{j}=0$ then

6: $\quad h_{j+1}=\left(\frac{\left|h_{j}\right|}{2}\right)^{\frac{1}{2}}$

7: if $g_{j}>0$ then

8: $\quad g_{j+1}=\frac{h_{j}}{2 h_{j+1}}$

9: $\quad$ end if

10: $\quad$ if $g_{j}<0$ then

11: $\quad h_{j+1}=0$

12: $\quad$ end if

13: end if

14: if $g_{j}>0$ then

15: $\quad h_{j+1}=\left(-\frac{g_{j}}{2}+\left[g_{j}^{2}+h_{j}^{2}\right]^{\frac{1}{2}}\right)^{\frac{1}{2}}$

16: $\quad g_{j+1}=\frac{h_{j}}{2 h_{j+1}}$

17: $\quad$ if $h_{j}<0$ then

18: $\quad h_{j+1}=-h_{j+1}$

19: end if

20: end if

where $m, l, j$ are natural numbers and $\theta, \gamma, \beta, \lambda$ real numbers. This system of $m$ equations comprises different types of transformations $\left(B_{k}\right)$ and processes $\left(K_{k}\right)$, namely and $l$ transformations and $m-l$ processes with many iterations starting at $\left(g_{0}, h_{0}\right)$. Applying this process to the system (2.1) transforms it into the discredited system (at the iteration $j$ ) given by

$$
\frac{d X_{j}}{d t}=M_{1}\left[X_{j}-s\left({ }^{T} M_{2} X_{j}-4\right) M_{3}\right]
$$

with $X_{j}=X_{j}(t)=\left(x_{j}(t), y_{j}(t), z_{j}(t)\right)^{T}$. Let us now associate to the process (3.2) the transformation $\mathbb{S}$ defined as

$$
\mathbb{S} \begin{cases}\mathbb{B}_{0}=g_{0}+h_{0}, \\ \mathbb{B}_{j+1}=g_{j+1}+h_{j+1}=\mathbb{B}_{1}\left[\left(\theta g_{j}+\gamma\right)+\left(\beta h_{j}+\lambda\right)\right], \\ \mathbb{B}_{j+2}=g_{j+2}+h_{j+2}=\mathbb{B}_{2}\left(g_{j+1}+h_{j+1}\right), \\ \mathbb{B}_{j+3}=g_{j+3}+h_{j+3}=\mathbb{K}_{1}\left(g_{j+2}+h_{j+2}\right), \\ \mathbb{B}_{j+4}=g_{j+4}+h_{j+4}=\mathbb{B}_{3}\left(g_{j+3}+h_{j+3}\right), \\ & \vdots \\ \mathbb{B}_{k+1}=g_{k+1}+h_{k+1} & =\mathbb{K}_{l}\left(g_{k-1}+h_{k-1}\right), \\ & \vdots \\ \mathbb{B}_{k+1}=g_{m}+h_{m} & =\mathbb{B}_{m-l}\left(g_{m-1}+h_{m-1}\right) .\end{cases}
$$

We can now use the so-called partial composition applicable in multivariate functions. 
Definition 1. Let $B$ be a multivariate function defined on $\mathbb{C}^{n}$ as

$$
B: \mathbb{C}^{n} \longrightarrow \mathbb{C}^{n}, \quad\left(g_{1}, g_{2}, \cdots, h_{n}\right) \longmapsto B\left(g_{1}, g_{2}, \cdots, h_{n}\right)
$$

and $g$ defined as

$$
g: \mathbb{C}^{n} \longrightarrow \mathbb{C}, \quad\left(g_{1}, g_{2}, \cdots, g_{n}\right) \longmapsto g\left(g_{1}, g_{2}, \cdots, g_{n}\right),
$$

the partial composition $\left.B\right|_{g_{j}=g}$ of $B$ and $g$ is the function obtained when the function $g$ takes the place of an argument $g_{j}$ of $B$ leading to

$$
\left.B\right|_{g_{j}=g}=B\left(g_{1}, \cdots, g_{j-1}, g\left(g_{1}, g_{2}, \cdots, h_{n}\right), g_{j+1}, \cdots, g_{n}\right) .
$$

If the function $g$ is a constant $g_{0} \in \mathbb{C}$ the partial composition becomes

$$
\left.B\right|_{g_{j}=g}=B\left(g_{1}, \cdots, g_{j-1}, g_{0}, \cdots, g_{n}\right) .
$$

We can now defined the modified fractal processes system $\tilde{\Psi}$ that takes the iterative representation:

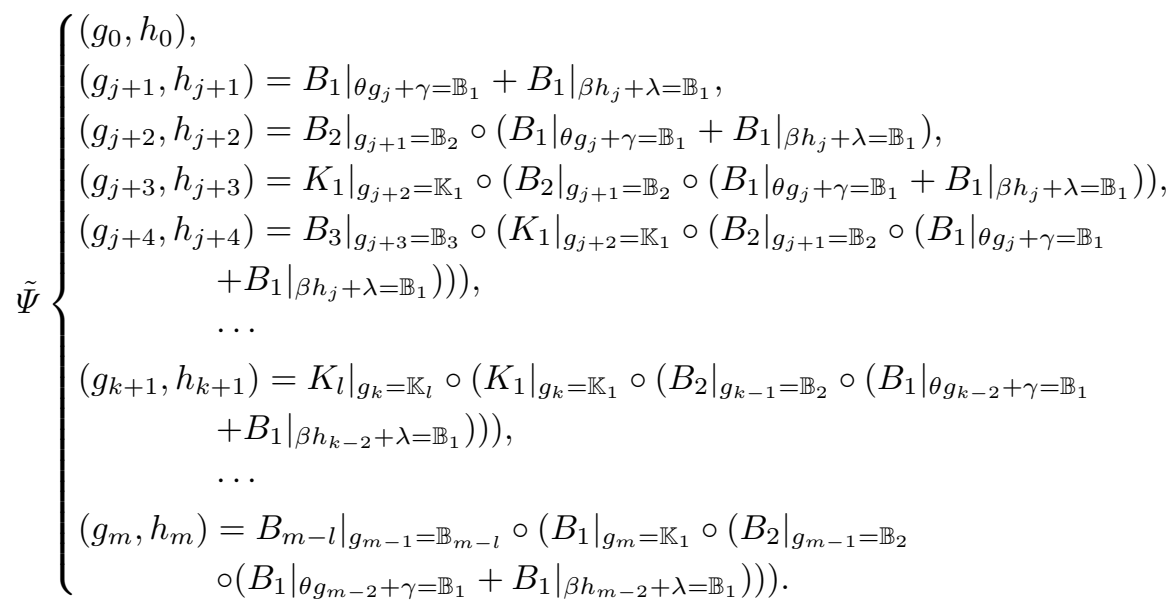

Taking the parameter value $d=0$ for two consecutive iterations $I_{j}$ and $I_{j+1}$, and taking $K_{j}$ as

$$
K_{j}=\left(\begin{array}{cc}
-g_{j} \times\left(1-g_{j}\right) & h_{1} \times\left(1-h_{j}\right) \\
h_{j} \times\left(1-h_{j}\right) & g_{1} \times\left(1-g_{j}\right)
\end{array}\right),
$$

and taking $m=6, \tilde{\Psi}$ becomes

$$
\tilde{\Psi}\left\{\begin{aligned}
\left(g_{1}, h_{1}\right)=\left.B_{1}\right|_{\theta g_{\gamma}=\mathbb{B}_{1}}+\left.B_{1}\right|_{\beta h_{\lambda}=\mathbb{B}_{1}}, \\
\left(g_{2}, h_{2}\right)=\left.B_{2}\right|_{g_{1}=\mathbb{B}_{2}} \circ\left(\left.B_{1}\right|_{\theta g_{\gamma}=\mathbb{B}_{1}}+\left.B_{1}\right|_{\beta h_{\lambda}=\mathbb{B}_{1}}\right), \\
\left(g_{3}, h_{3}\right)=\left.K_{1}\right|_{g_{2}=\mathbb{K}_{1}} \circ\left(\left.B_{2}\right|_{g_{1}=\mathbb{B}_{2}} \circ\left(\left.B_{1}\right|_{\theta g_{\gamma}=\mathbb{B}_{1}}+\left.B_{1}\right|_{\beta h_{\lambda}=\mathbb{B}_{1}}\right)\right), \\
\left(g_{4}, h_{4}\right)=\left.B_{3}\right|_{g_{3}=\mathbb{B}_{3}} \circ\left(\left.K_{1}\right|_{g_{2}=\mathbb{K}_{1}} \circ\left(\left.B_{2}\right|_{g_{1}=\mathbb{B}_{2}} \circ\left(\left.B_{1}\right|_{\theta g_{\gamma}=\mathbb{B}_{1}}+\left.B_{1}\right|_{\beta h_{\lambda}=\mathbb{B}_{1}}\right)\right)\right), \\
\left(g_{5}, h_{5}\right)=\left.B_{4}\right|_{g_{4}=\mathbb{B}_{4}} \circ\left(B _ { 3 } | _ { g _ { 3 } = \mathbb { B } _ { 3 } } \circ \left(K _ { 1 } | _ { g _ { 2 } = \mathbb { K } _ { 1 } } \circ \left(B _ { 2 } | _ { g _ { 1 } = \mathbb { B } _ { 2 } } \circ \left(\left.B_{1}\right|_{\theta g_{\gamma}=\mathbb{B}_{1}}\right.\right.\right.\right. \\
\left.\left.\left.+\left.B_{1}\right|_{\beta h_{\lambda}=\mathbb{B}_{1}}\right)\right)\right), \\
\left(g_{6}, h_{6}\right)=\left.B_{5}\right|_{g_{5}=\mathbb{B}_{5}} \circ\left(B _ { 4 } | _ { g _ { 4 } = \mathbb { B } _ { 4 } } \circ \left(B _ { 3 } | _ { g _ { 3 } = \mathbb { B } _ { 3 } } \circ \left(K _ { 1 } | _ { g _ { 2 } = \mathbb { K } _ { 1 } } \circ \left(\left.B_{2}\right|_{g_{1}=\mathbb{B}_{2}}\right.\right.\right.\right. \\
\left.\left.\left.\circ\left(\left.B_{1}\right|_{\theta g_{\gamma}=\mathbb{B}_{1}}+\left.B_{1}\right|_{\beta h_{\lambda}=\mathbb{B}_{1}}\right)\right)\right)\right) .
\end{aligned}\right.
$$


The implementation of this scheme with $g_{k}=x_{j+1}-x_{j}, \quad h_{k}=y_{j+1}-y_{j}, \quad k, j=$ $1,2, \ldots, 3$, depicts the illustration shown in Figure 8.

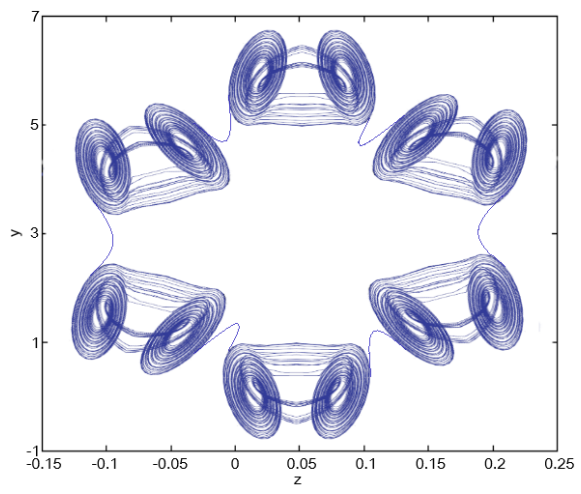

Figure 8. Self-similarity dynamic for the chaotic attractor with two scrolls generated by the model (3.3) using the Fractal (Julia's) process with $d=0$. We can see a set of six similar double-scroll chaotic attractors grouped to form a symmetry around the axis $z=0.05$.

It shows the elf-similarity dynamic for the chaotic attractor with two scrolls generated by the model (3.3) using the Fractal (Julia's) process with $d=0$. We can see a set of six similar double-scroll chaotic attractors grouped to form a symetry aroud the axis $z=0.05$.

Similarly, we apply the above process to the system (2.2) and transform it into the discredited system (at the iteration $j$ ) given by

$$
\frac{d X_{j}}{d t}=\tilde{M}_{1}\left[X_{j}-s\left({ }^{T} \tilde{M}_{2} X_{j}-4\right) \tilde{M}_{3}\right]
$$

with

$$
\tilde{M}_{1}=\left(\begin{array}{ccc}
a & c & 0 \\
-c & a & 0 \\
0 & 0 & -e
\end{array}\right), \quad \tilde{M}_{2}={ }^{T}(0, f, f) \text { and } \tilde{M}_{3}={ }^{T}(0,1,1)
$$

with the constants taken for two consecutive iterations $I_{j}$ and $I_{j+1}$ as in Table 1.

Table 1. Parameter values.

\begin{tabular}{lccccccccccccc}
\hline & $b_{1}$ & $b_{2}$ & $b_{3}$ & $b_{4}$ & $s_{1}$ & $s_{2}$ & $s_{3}$ & $s_{4}$ & $s_{5}$ & $a$ & $c$ & $e$ & $f$ \\
\hline$I_{j}$ & $\frac{-1}{4}$ & $\frac{1}{4}$ & $\frac{3}{4}$ & $\frac{5}{4}$ & $\frac{-1}{2}$ & 0 & $\frac{1}{2}$ & 1 & $\frac{3}{2}$ & $\frac{1}{4}$ & 5 & $\frac{5}{2}$ & $\frac{1}{4}$ \\
$I_{j+1}$ & $\frac{-1}{8}$ & $\frac{1}{8}$ & $\frac{3}{8}$ & $\frac{5}{8}$ & $\frac{-1}{4}$ & 0 & $\frac{1}{4}$ & $\frac{1}{2}$ & $\frac{3}{4}$ & $\frac{1}{8}$ & $\frac{5}{2}$ & $\frac{5}{4}$ & $\frac{1}{8}$ \\
\hline
\end{tabular}

Then, we have the implementation of this scheme with $g_{k}=x_{j+1}-x_{j}, \quad h_{k}=$ $y_{j+1}-y_{j}, \quad k, j=1,2, \ldots, 3$, that generates the illustration shown in Figure 9 . 
It shows self-similarity dynamic for the chaotic attractor with five scrolls generated by the model (3.4) using the Fractal (Julia's) process with the parameter values shown in Table 1 . We can see a set of six similar quintuple-scroll chaotic attractors grouped to form a symmetry around the axis $y=3$.

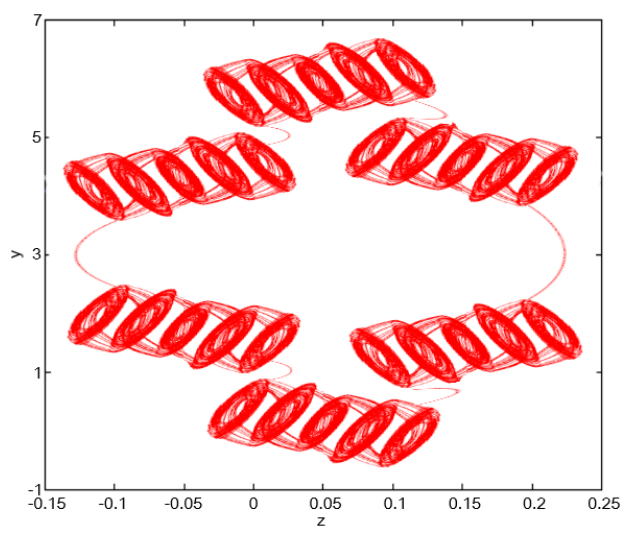

Figure 9. Self-similarity dynamic for the chaotic attractor with five scrolls generated by the model (3.4) using the Fractal (Julia's) process with the parameter values shown in Table 1. We can see a set of six similar quintuple-scroll chaotic attractors grouped to form a symmetry around the axis $y=3$.

\section{Fractal-fractional process in self-similarity for attractors with many scrolls}

We continue the analysis in this section by applying the fractal operator combined with the fractional dynamics that gives the so-called fractal-fractional operator defined as follows $[2,3,8,9]$ :

Definition 2. Let $X \in A \subseteq \mathbb{R}^{3}$, and $a_{0}, b_{0} \in \mathbb{R}$. We assume that the function $w(X, t)$ defined on $A \times\left(a_{0}, b_{0}\right)$ is fractal differentiable with respect to the $t$-variable on $\left(a_{0}, b_{0}\right)$ with order $\sigma$ then, the Riemann-Liouville sense fractalfractional derivative of $w$ with order $\sigma$ and power law is defined as:

$$
{ }^{F F} D_{t}^{\sigma} w(X, t)=\frac{1}{\Gamma(1-\sigma)} \frac{\partial}{\partial t^{\sigma}} \int_{0}^{t} w(X, \zeta)(t-\zeta)^{-\sigma} d \zeta
$$

where $\frac{\partial}{\partial t^{\sigma}} w$ is defined as

$$
\frac{\partial}{\partial t^{\sigma}} w\left(X, t_{0}\right)=\lim _{t \rightarrow t_{0}} \frac{w(X, t)-w\left(X, t_{0}\right)}{t^{\sigma}-t_{0}^{\sigma}} .
$$

The general version of that definition is given by

$$
{ }^{F F} D_{t}^{\sigma, \varsigma} w(X, t)=\frac{1}{\Gamma(1-\sigma)} \frac{\partial^{\varsigma}}{\partial t^{\sigma}} \int_{0}^{t} w(X, \zeta)(t-\zeta)^{-\sigma} d \zeta
$$


with $\varsigma>0$ and $\frac{\partial^{\varsigma}}{\partial t^{\sigma}} w$ given by

$$
\frac{\partial^{\varsigma}}{\partial t^{\sigma}} w\left(X, t_{0}\right)=\lim _{t \rightarrow t_{0}} \frac{w^{\varsigma}(X, t)-w^{\varsigma}\left(X, t_{0}\right)}{t^{\sigma}-t_{0}^{\sigma}} .
$$

We associate to that fractal-fractional operator (4.1), its anti-derivative also known as the fractal-fractional integral of same order $\sigma$, very important in solving systems of fractional differential equations and defined by:

$$
{ }^{F F} \mathbf{I}_{t}^{\sigma} w(t)=\frac{\sigma}{\Gamma(\sigma)} \int_{0}^{t} \zeta^{-\sigma} w(\zeta)(t-\zeta)^{\sigma-1} d \zeta, \quad t>0
$$

The application of the fractal-fractional operator (4.1) to the system of equations (2.1) yields

$$
{ }^{F F} D_{t}^{\sigma} X(t)=M_{1}\left[X-s\left({ }^{T} M_{2} X-4\right) M_{3}\right],
$$

where we recall that

$$
\begin{aligned}
& X=X(t)=\left(\begin{array}{l}
x(t) \\
y(t) \\
z(t)
\end{array}\right), \quad s(\zeta)=\left\{\begin{array}{ll}
1, & \text { when } \zeta>0, \\
0, & \text { when } \zeta \leq 0
\end{array},\right. \\
& M_{1}=\left(\begin{array}{ccc}
\frac{1}{2} & 10 & 0 \\
-10 & \frac{1}{2} & 0 \\
0 & 0 & -10
\end{array}\right), \quad M_{2}={ }^{T}(d, 4,4) \text { and } M_{3}={ }^{T}(0,1,1) \text {. }
\end{aligned}
$$

In order to adequately solve this combined system, we are forced to add the initial conditions given as follows:

$$
x(0)=\mathbf{x}(x), y(0)=\mathbf{y}(y), z(0)=\mathbf{z}(z) .
$$

Making use of the Legendre wavelets method as proposed in [5,20], we can go on now by transforming the system (4.3)-(4.4) into a compact form. Using now the associated antiderivative (4.2) and applying on both sides of the model (4.3) leads to

$$
\left\{\begin{array}{l}
F F D_{t}^{\sigma} x(t)={ }^{T} P_{r}^{1} \Phi_{r}(t) \\
F F D_{t}^{\sigma} y(t)={ }^{T} P_{r}^{2} \Phi_{r}(t) \\
F F D_{t}^{\sigma} z(t)={ }^{T} P_{r}^{3} \Phi_{r}(t)
\end{array}\right.
$$

Here $\Phi_{r}(t)$ is the matrix whose elements define the Legendre wavelets given as

$$
\varphi_{n r}(t)= \begin{cases}\left.2^{m / 2} \sqrt{2 r+1} \mathcal{L}_{r}^{*}\left(2^{m} t-n\right)\right), & \text { if } t \in\left[\frac{n}{2^{m}}, \frac{n+1}{2^{m}}\right] \\ 0, & \text { elsewhere }\end{cases}
$$

with $\mathcal{L}_{r}^{*}$ the shifted Legendre polynomial defined on $[0,1]$ as $\mathcal{L}_{r}^{*}(t)=\mathcal{L}_{r}(2 t-1)$, $\left(\mathcal{L}_{r}(2 t-1)\right)_{r}$ being the family

$$
\mathcal{L}_{0}=1, \quad \mathcal{L}_{1}=x, \quad \mathcal{L}_{r+1}(x)=\frac{1+2 r}{r+1} x \mathcal{L}_{r}(x)-\frac{r}{1+r} \mathcal{L}_{r-1}(x), \quad r=1,2, \ldots, N-1 .
$$


Moreover, $N$ a positive integer number, $n=1,2, \ldots, 2^{m}-1$ and $m=0,1,2, \ldots$. $P_{r}^{i}={ }^{T}\left[\mathbf{r}_{r}^{1}, \mathbf{r}_{r}^{2}, \ldots, \mathbf{r}_{r}^{r}\right]$ are coefficients to be found with ${ }^{T} M_{r}^{1},{ }^{T} M_{r}^{2}$ and ${ }^{T} M_{r}^{3}$ are the transpose of the matrices $M_{r}^{1}, M_{r}^{2}$ and $M_{r}^{3}$ respectively. Associating the initial conditions yields

$$
\left\{\begin{array}{l}
x(t) \approx{ }^{T} P_{r}^{1} Q_{r \times r}^{\sigma} \Phi_{r}(t)+\mathbf{x} \\
y(t) \approx P_{r}^{2} Q_{r \times r}^{\sigma} \Phi_{r}(t)+\mathbf{y} \\
z(t) \approx P_{r}^{3} Q_{r \times r}^{\sigma} \Phi_{r}(t)+\mathbf{z}
\end{array}\right.
$$

where $Q_{r \times r}^{\sigma}$ is the Legendre operational matrix of integration and the subscript $r$ denotes its dimension. We know that [5,20], Legendre wavelets can be expanded into an $r$-term form as

$$
\Phi_{r}(t)=\Xi_{r \times r} A_{r}(t),
$$

where $A_{r}(t)={ }^{T}\left[a_{1}(t), a_{2}(t), \ldots, a_{r}(t)\right]$ is the Block Pulse Functions so that

$$
a_{l}(t)= \begin{cases}1, & \text { if } t \in\left[\frac{m-1}{r}, \frac{m}{r}\right] ; \\ 0, & \text { elsewhere }\end{cases}
$$

for each $m=1,2, \ldots r$, and $\Xi$ the Legendre wavelet matrix

$$
\Xi_{r \times r}=\left[\Phi_{r}\left(\frac{1}{2 r}\right) \Phi_{r}\left(\frac{3}{2 r}\right) \cdots \Phi_{r}\left(\frac{2 r-1}{2 r}\right)\right] .
$$

Now the substitution of (4.7) into system (4.6) leads to

$$
\left\{\begin{array}{l}
x(t) \approx{ }^{T} P_{r}^{1} Q_{r \times r}^{\sigma} \Xi_{r \times r} A_{r}(t)+\left[\mathbf{x}_{i}\right] A_{r}(t), \\
y(t) \approx{ }^{T} P_{r}^{2} Q_{r \times r}^{\sigma} \Xi_{r \times r} A_{r}(t)+\left[\mathbf{y}_{i}\right] A_{r}(t), \\
z(t) \approx{ }^{T} P_{r}^{3} Q_{r \times r}^{\sigma} \Xi_{r \times r} A_{r}(t)+\left[\mathbf{z}_{i}\right] A_{r}(t),
\end{array}\right.
$$

where

$$
\left[\mathbf{x}_{i}\right]=\left[\mathbf{x}_{1}, \mathbf{x}_{2}, \ldots, \mathbf{x}_{r}\right],\left[\mathbf{y}_{i}\right]=\left[\mathbf{y}_{1}, \mathbf{y}_{2}, \ldots, \mathbf{y}_{r}\right],\left[\mathbf{z}_{i}\right]=\left[\mathbf{z}_{1}, \mathbf{z}_{2}, \ldots, \mathbf{z}_{r}\right] .
$$

Now let

$$
{ }^{T} P_{r}^{i} Q_{r \times r}^{\sigma} \Xi_{r \times r}=\mathcal{M}_{1 \times r}^{\sigma, i}=\left[\mathbf{r}_{1}^{\sigma, i}, \mathbf{r}_{2}^{\sigma, i}, \ldots, \mathbf{r}_{r}^{\sigma, i}\right] .
$$

Now using the collocations points $t_{i}=\frac{2 i-1}{2^{m+1} N}, \quad i=1,2,3, \ldots, r, N \in \mathbb{N}$, to disperse $t$, the substitution of (4.8) and (4.9) into the system (4.3) and lead to

$$
\left\{\begin{aligned}
{ }^{T} P_{r}^{1} \Xi_{r \times r}= & b\left[\mathbf{r}_{1}^{\sigma, 2}, \mathbf{r}_{2}^{\sigma, 2}, \ldots, \mathbf{r}_{r}^{\sigma, 2}\right]+(a-p)\left[\mathbf{r}_{1}^{\sigma, 1}, \mathbf{r}_{2}^{\sigma, 1}, \ldots, \mathbf{r}_{r}^{\sigma, 1}\right] \\
& +\left[\mathbf{x}_{1}, \mathbf{x}_{2}, \ldots, \mathbf{x}_{r}\right] \\
{ }^{T} P_{r}^{2} \Xi_{r \times r}= & a\left[\mathbf{r}_{1}^{\sigma, 1}, \mathbf{r}_{2}^{\sigma, 1}, \ldots, \mathbf{r}_{r}^{\sigma, 1}\right]-(b+p)\left[\mathbf{r}_{1}^{\sigma, 2}, \mathbf{r}_{2}^{\sigma, 2}, \ldots, \mathbf{r}_{r}^{\sigma, 2}\right] \\
& +\left[\mathbf{y}_{1}, \mathbf{y}_{2}, \ldots, \mathbf{y}_{r}\right] \\
{ }^{T} P_{r}^{3} \Xi_{r \times r}= & p e_{1}[1,1 \ldots, 1]+c\left[\mathbf{r}_{1}^{\sigma, 3}, \mathbf{r}_{2}^{\sigma, 3}, \ldots, \mathbf{r}_{r}^{\sigma, 3}\right] \\
& +\left[\mathbf{z}_{1}, \mathbf{z}_{2}, \ldots, \mathbf{z}_{r}\right]
\end{aligned}\right.
$$

Hence, we obtain this non-linear system equations with $3 r$ unknown coefficients, leading to the $1 \times 3 r$ unknown coefficients $\mathbf{r}_{m}^{\sigma, i}$ of the matrix

$$
\mathcal{M}_{3 \times r}^{\sigma}={ }^{T}\left(\mathcal{M}_{1 \times r}^{\sigma, 1}, \mathcal{M}_{1 \times r}^{\sigma, 2}, \mathcal{M}_{1 \times r}^{\sigma, 3}\right)=\left(\begin{array}{c}
\mathbf{r}_{1}^{\sigma, 1}, \mathbf{r}_{2}^{\sigma, 1}, \ldots, \mathbf{r}_{r}^{\sigma, 1} \\
\mathbf{r}_{1}^{\sigma, 2}, \mathbf{r}_{2}^{\sigma, 2}, \ldots, \mathbf{r}_{r}^{\sigma, 2} \\
\mathbf{r}_{1}^{\sigma, 3}, \mathbf{r}_{2}^{\sigma, 3}, \ldots, \mathbf{r}_{r}^{\sigma, 3}
\end{array}\right),
$$

which unknown coefficients are easily found using Newton iteration method. Then exploiting (4.6), leads the sought numerical solution $(x(t), y(t), z(t))$. 


\subsection{Error analysis}

We assume here that the solution $X(t)=(x(t), y(t), z(t))$ is a function whose second order derivative is bounded as $\left|X^{\prime \prime}(t)\right| \leq \theta_{0}$, equivalently

$$
\left|x^{\prime \prime}(t)\right| \leq \theta_{0}^{1},\left|y^{\prime \prime}(t)\right| \leq \theta_{0}^{1}\left|z^{\prime \prime}(t)\right| \leq \theta_{0}^{1} .
$$

Making use of the Legendre wavelets schemes described here above to approximate the solution $X(t)$ means it can be expanded as a uniformly convergent series that reads as

$$
x(t)=\sum_{n=0}^{\infty} \sum_{r=0}^{\infty} \mathbf{r}_{n r}^{\sigma, 1} \varphi_{n r}(t), \quad y(t)=\sum_{n=0}^{\infty} \sum_{r=0}^{\infty} \mathbf{r}_{n r}^{\sigma, 2} \varphi_{n r}(t), \quad z(t)=\sum_{n=0}^{\infty} \sum_{r=0}^{\infty} \mathbf{r}_{n r}^{\sigma, 2} \varphi_{n r}(t)
$$

with

$$
\mathbf{r}_{n r}^{\sigma, 1}=\left\langle x(t), \varphi_{n r}(t)\right\rangle, \mathbf{r}_{n r}^{\sigma, 2}=\left\langle y(t), \varphi_{n r}(t)\right\rangle, \mathbf{r}_{n r}^{\sigma, 3}=\left\langle z(t), \varphi_{n r}(t)\right\rangle .
$$

We have the following convergence results.

Proposition 1. Let $\theta_{0}^{i}>0, i=1,2,3$. Assuming that the solution $(x(t), y(t), z(t))$ is made of continuous functions on $[0,1]$ whose second order derivatives are bounded as

$$
\left|x^{\prime \prime}(t)\right| \leq \theta_{0}^{1}, \quad\left|y^{\prime \prime}(t)\right| \leq \theta_{0}^{2} \quad\left|z^{\prime \prime}(t)\right| \leq \theta_{0}^{3},
$$

then the coefficients $\boldsymbol{r}_{n r}^{\sigma, i}, i=1,2,3$, satisfy

$$
\left|\boldsymbol{r}_{n r}^{\sigma, 1}\right|<\frac{(12)^{1 / 2} \theta_{0}^{1}}{(2 r-3)^{2}(\sqrt{2 n})^{5}},\left|\boldsymbol{r}_{n r}^{\sigma, 2}\right|<\frac{(12)^{1 / 2} \theta_{0}^{2}}{(2 r-3)^{2}(\sqrt{2 n})^{5}},\left|\boldsymbol{r}_{n r}^{\sigma, 3}\right|<\frac{(12)^{1 / 2} \theta_{0}^{3}}{(2 r-3)^{2}(\sqrt{2 n})^{5}}
$$

Proof. Let us start with the function $x(t)$ and using definition the Legendre wavelets coefficients given in (4.10) and taking into account (4.5) we have

$$
\begin{aligned}
& \mathbf{r}_{n r}^{\sigma, 1}=\int_{0}^{1} x(t) \varphi_{n r}(t) d t \\
& =\int_{\frac{n}{2^{m}}}^{\frac{n+1}{2^{m}}} x(t) 2^{m / 2} \sqrt{2 r+1} \mathcal{L}_{r}^{*}\left(2^{m} t-n\right) d t=\sqrt{\frac{1+2 r}{2^{m}}} \int_{0}^{1} x\left(\frac{n+\delta}{2^{m}}\right) \mathcal{L}_{r}^{*}(\delta) d \delta,
\end{aligned}
$$

where we have changed the variable as $t=\frac{n+\delta}{2^{m}}$

$$
\begin{aligned}
& =\sqrt{\frac{1}{(2 r+1) 2^{3 m+2}}} \int_{0}^{1} \frac{d x}{d t}\left(\frac{n+\delta}{2^{m}}\right)\left(\mathcal{L}_{r+1}^{*}(\delta)-\mathcal{L}_{r-1}^{*}(\delta)\right) d \delta \\
& =\sqrt{\frac{1}{(2 r+1) 2^{5 m+2}}} \int_{0}^{1} \frac{d^{2} x}{d t^{2}}\left(\frac{n+\delta}{2^{m}}\right)\left(\frac{\mathcal{L}_{r+2}^{*}(\delta)-\mathcal{L}_{r}^{*}(\delta)}{6+4 r}-\frac{\mathcal{L}_{r}^{*}(\delta)-\mathcal{L}_{r-2}^{*}(\delta)}{-2+4 r}\right) d \delta,
\end{aligned}
$$

where we have used the derivative properties of the shifted Legendre polynomials $[5,20]$. Hence

$$
\left|\mathbf{r}_{n r}^{\sigma, 1}\right| \leq\left|\sqrt{\frac{1}{(2 r+1) 2^{5 m+2}}}\right| \int_{0}^{1}\left|\frac{d^{2} x}{d t^{2}}\left(\frac{n+\delta}{2^{m}}\right)\right|
$$




$$
\times\left|\left(\frac{\mathcal{L}_{r+2}^{*}(\delta)-\mathcal{L}_{r}^{*}(\delta)}{6+4 r}-\frac{\mathcal{L}_{r}^{*}(\delta)-\mathcal{L}_{r-2}^{*}(\delta)}{-2+4 r}\right)\right| d \delta .
$$

After developing the right hand side of inequality and making use of the constraint property $\left|x^{\prime \prime}(t)\right| \leq \theta_{0}^{1}$ and the orthogonality property of the shifted Legendre polynomials finally leads to

$$
\left|\mathbf{r}_{n r}^{\sigma, 1}\right| \leq \sqrt{\frac{1}{\sqrt{2 r+1}}} \frac{1}{2^{(5 / 2) m+1}} \theta_{0}^{1} \sqrt{\frac{\sqrt{3}}{(2 r-3)}} \frac{1}{2 r-1}<\frac{(12)^{1 / 2} \theta_{0}^{1}}{(2 r-3)^{2}(\sqrt{2 n})^{5}} .
$$

We do the similar analysis for the functions $y(t)$ and $z(t)$ and the proposition is concluded.

This result leads to the following error estimate:

Corollary 1. Let $\theta_{0}^{i}>0, i=1,2,3$. Assuming that the solution $(x(t), y(t), z(t))$ is made of continuous functions on $[0,1]$ whose second order derivatives are bounded as

$$
\left|x^{\prime \prime}(t)\right| \leq \theta_{0}^{1}, \quad\left|y^{\prime \prime}(t)\right| \leq \theta_{0}^{2}, \quad\left|z^{\prime \prime}(t)\right| \leq \theta_{0}^{3},
$$

then the error made when $x_{k N}=\sum_{n=0}^{2^{m}-1} \sum_{r=0}^{N-1} \mathbf{r}_{n r}^{\sigma, 1} \varphi_{n r}(t)$ approximates $x(t)$ satisfies

$$
\Delta_{k N}^{1}<(12)^{1 / 2} \theta_{0}^{1} \sqrt{\sum_{n=2^{m}}^{\infty} \sum_{r=N}^{\infty} \frac{1}{32 n^{5}(2 r-3)^{4}}}
$$

Table 2. Errors made when using the Legendre wavelets for the model (4.3).

\begin{tabular}{lllllll}
\hline \multicolumn{3}{c}{$\sigma=1$} & & \multicolumn{3}{c}{$\sigma=0.9$} \\
\hline$t$ & $\left(\Delta^{1}\right)_{x(t)}$ & $\left(\Delta^{2}\right)_{y(t)}$ & $\left(\Delta^{3}\right)_{z(t)}$ & $\left(\Delta^{1}\right)_{x(t)}$ & $\left(\Delta^{2}\right)_{y(t)}$ & $\left(\Delta^{3}\right)_{z(t)}$ \\
\hline 0.1 & $2.342 \mathrm{e}^{-4}$ & $1.276 \mathrm{e}^{-4}$ & $3.562 \mathrm{e}^{-3}$ & $2.672 \mathrm{e}^{-4}$ & $3.577 \mathrm{e}^{-4}$ & $3.044 \mathrm{e}^{-3}$ \\
0.2 & $4.245 \mathrm{e}^{-4}$ & $1.287 \mathrm{e}^{-5}$ & $2.245 \mathrm{e}^{-4}$ & $6.782 \mathrm{e}^{-4}$ & $0.266 \mathrm{e}^{-3}$ & $7.244 \mathrm{e}^{-4}$ \\
0.3 & $5.167 \mathrm{e}^{-4}$ & $2.298 \mathrm{e}^{-4}$ & $6.234 \mathrm{e}^{-2}$ & $3.892 \mathrm{e}^{-4}$ & $0.552 \mathrm{e}^{-4}$ & $3.332 \mathrm{e}^{-4}$ \\
0.4 & $2.896 \mathrm{e}^{-5}$ & $3.109 \mathrm{e}^{-5}$ & $5.223 \mathrm{e}^{-4}$ & $2.902 \mathrm{e}^{-3}$ & $5.446 \mathrm{e}^{-4}$ & $3.332 \mathrm{e}^{-4}$ \\
0.6 & $3.201 \mathrm{e}^{-4}$ & $2.900 \mathrm{e}^{-3}$ & $3.212 \mathrm{e}^{-3}$ & $1.092 \mathrm{e}^{-5}$ & $3.332 \mathrm{e}^{-5}$ & $4.332 \mathrm{e}^{-4}$ \\
0.7 & $2.223 \mathrm{e}^{-3}$ & $4.782 \mathrm{e}^{-3}$ & $2.221 \mathrm{e}^{-4}$ & $3.985 \mathrm{e}^{-4}$ & $5.522 \mathrm{e}^{-5}$ & $3.442 \mathrm{e}^{-5}$ \\
0.8 & $2.245 \mathrm{e}^{-4}$ & $4.562 \mathrm{e}^{-4}$ & $4.643 \mathrm{e}^{-3}$ & $3.872 \mathrm{e}^{-5}$ & $3.112 \mathrm{e}^{-5}$ & $3.244 \mathrm{e}^{-4}$ \\
0.9 & $1.267 \mathrm{e}^{-3}$ & $4.342 \mathrm{e}^{-3}$ & $0.254 \mathrm{e}^{-4}$ & $3.787 \mathrm{e}^{-5}$ & $3.722 \mathrm{e}^{-3}$ & $4.266 \mathrm{e}^{-4}$ \\
1 & $2.892 \mathrm{e}^{-5}$ & $3.022 \mathrm{e}^{-4}$ & $3.265 \mathrm{e}^{-3}$ & $4.988 \mathrm{e}^{-5}$ & $2.349 \mathrm{e}^{-3}$ & $3.235 \mathrm{e}^{-4}$ \\
\hline
\end{tabular}

Similarly, the error made when $y_{k N}=\sum_{n=0}^{2^{m}-1} \sum_{r=0}^{N-1} \mathbf{r}_{n r}^{\sigma, 2} \varphi_{n r}(t)$ and $z_{k N}=$ $\sum_{n=0}^{2^{m}-1} \sum_{r=0}^{N-1} \mathbf{r}_{n r}^{\sigma, 3} \varphi_{n r}(t)$ respectively approximate $y(t)$ and $z(t)$ satisfy

$$
\begin{aligned}
& \Delta_{k N}^{2}<(12)^{1 / 2} \theta_{0}^{2} \sqrt{\sum_{n=2^{m}}^{\infty} \sum_{r=N}^{\infty} \frac{1}{32 n^{5}(2 r-3)^{4}}}, \\
& \Delta_{k N}^{3}<(12)^{1 / 2} \theta_{0}^{3} \sqrt{\sum_{n=2^{m}}^{\infty} \sum_{r=N}^{\infty} \frac{1}{32 n^{5}(2 r-3)^{4}}} .
\end{aligned}
$$




\section{$5 \quad$ Numerical simulations}

We can now implement the above scheme using the parameter values given by $d=0$. Hence, for $m=2, N=3$ and $\sigma=1(\sigma=0.9)$, the errors made when approximating are in the Table 2 .

The implementation of this Legendre wavelets scheme generates the illustration shown in Figures 10-12 depicting the self-similarity process for the chaotic attractor with two scrolls generated by the model (4.3)-(4.4) using the using the Legendre wavelets scheme with $d=0$ and for $\sigma=1, \sigma=0.9$ and $\sigma=0.8$ respectively.

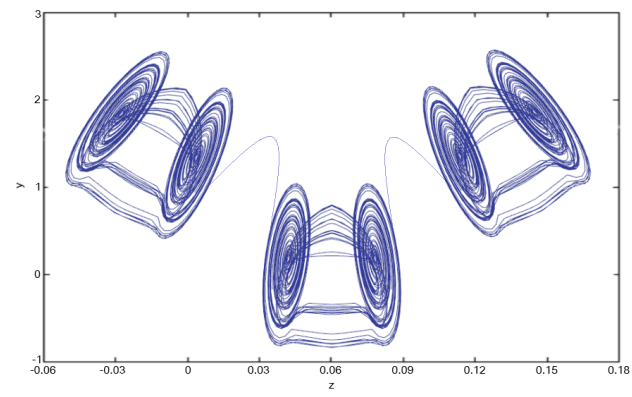

Figure 10. Self-similarity dynamic for the chaotic attractor with two scrolls generated by the model (4.3)-(4.4) using the using the Legendre wavelets scheme with $d=0$ and $\sigma=1$.

We can see a set of three similar double-scroll chaotic attractors grouped to form a symmetry around the axis $z=0.06$.

The simulations show a set of three similar double-scroll chaotic attractors grouped to form a symmetry around the axis $z=0.06$. In Figure 11, the two scrolls seem to move way from each other compared to those of Figure 10.

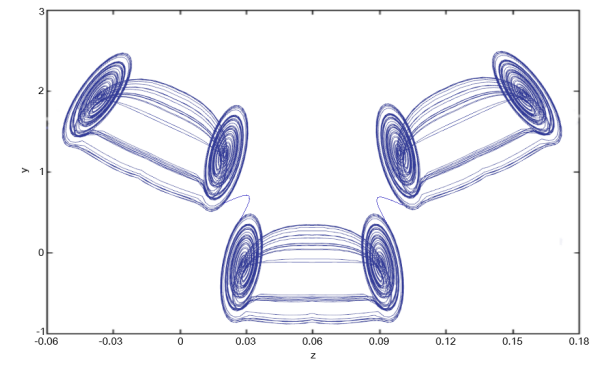

Figure 11. Self-similarity dynamic for the chaotic attractor with two scrolls generated by the model (4.3)-(4.4) using the using the Legendre wavelets scheme with $d=0$ and $\sigma=0.9$.

We can see a set of three similar double-scroll chaotic attractors grouped to form a symmetry around the axis $z=0.06$. The two scrolls seem to move way from each other compared to Figure 10.

Such a distance is shown to increase in Figure 12 where the derivative order $\sigma$ has decreased to 0.8 . 
In the same way, we apply of the fractal-fractional operator (4.1) to the system of equations $(2.2)$ as

$$
{ }^{F F} D_{t}^{\sigma} X(t)=\tilde{M}_{1}\left[X-s\left({ }^{T} \tilde{M}_{2} X-4\right) \tilde{M}_{3}\right]
$$

where we recall

$$
\tilde{M}_{1}=\left(\begin{array}{ccc}
a & c & 0 \\
-c & a & 0 \\
0 & 0 & -e
\end{array}\right), \quad \tilde{M}_{2}={ }^{T}(0, f, f) \text { and } \tilde{M}_{3}={ }^{T}(0,1,1) .
$$

and implement the Legendre wavelets scheme described above. Making use the parameter values given by

$$
\begin{gathered}
\left(b_{1}, b_{2}, b_{3}, b_{4}\right)=\left(-\frac{1}{2}, \frac{1}{2}, \frac{3}{2}, \frac{5}{2}\right),\left(s_{1}, s_{2}, s_{3}, s_{4}, s_{5}\right)=(-1,0,1,2,3), \\
(a, c, e, f)=(1 / 2,10,5,1 / 2) .
\end{gathered}
$$

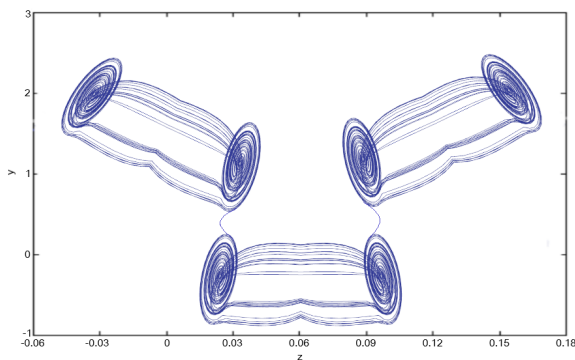

Figure 12. Self-similarity dynamic for the chaotic attractor with two scrolls generated by the model (4.3)-(4.4) using the using the Legendre wavelets scheme with $d=0$ and $\sigma=0.8$.

We can see a set of three similar double-scroll chaotic attractors grouped to form a symmetry around the axis $z=0.06$. The two scrolls seem to further move way from each other compared to Figure 11.

Hence, for $m=2, N=3$ and $\sigma=1$, we get the illustration shown in Figure 13. Lastly, we apply the fractal-fractional operator (4.1) to the system of equations (2.4) as

$$
\left\{\begin{array}{l}
F F D_{\tau}^{\sigma} x(\tau)=y-W(y+z), \\
F F D_{\tau}^{\sigma} y(\tau)=-x+b y+W(x+z)-b W(y+z), \\
F F D_{\tau}^{\sigma} z(\tau)=-z+d W(x+z)-d W(y+z),
\end{array}\right.
$$

where we recall $W(\eta)=0.5 \sum \frac{i=0}{n} v(\eta-i-0.5)$, where $v(\cdot)$ represents the unit step function and where we have set

$$
\tau=t / R C, R=3 k \Omega, \quad C=100 p F, \quad x=u_{1} / 1 V, \quad y=u_{2} / 1 V, \quad z=u_{3} / 1 V .
$$

We implement the Legendre wavelets scheme described above. Making use the parameter values given by

$$
(b, d, W(\eta))=\left(\frac{1}{10}, \frac{1}{2}, \frac{1}{2} v\left(\eta-\frac{1}{2}\right)+\frac{1}{2} v\left(\eta-\frac{3}{2}\right)\right) .
$$




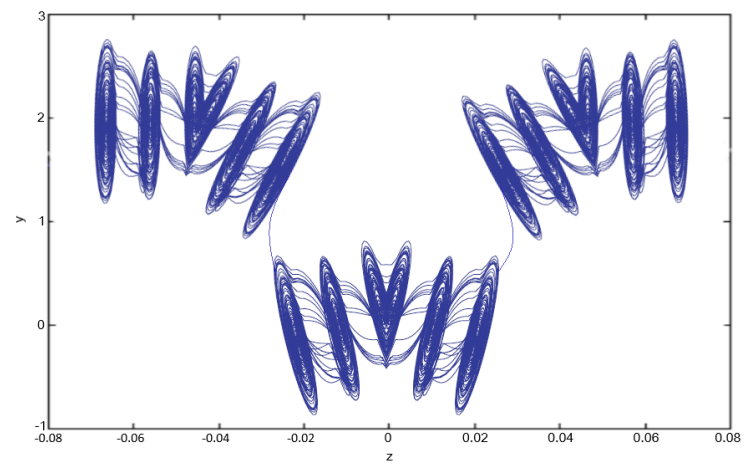

Figure 13. Self-similarity dynamic for the chaotic attractor with five scrolls generated by the model (5.1)-(4.4) using the using the Legendre wavelets scheme with the parameter values

$\left(b_{1}, b_{2}, b_{3}, b_{4}\right)=\left(-\frac{1}{2}, \frac{1}{2}, \frac{3}{2}, \frac{5}{2}\right),\left(s_{1}, s_{2}, s_{3}, s_{4}, s_{5}\right)=(-1,0,1,2,3),(a, c, e, f)=\left(\frac{1}{2}, 10,5, \frac{1}{2}\right)$, and $\sigma=1$. We can see a set of three similar quintuple-scroll chaotic attractors grouped to form a symmetry around the axis $z=0$.

Henceforth, the model (5.2) generates a chaotic attractor with seven scrolls, as depicted in Figure 14.

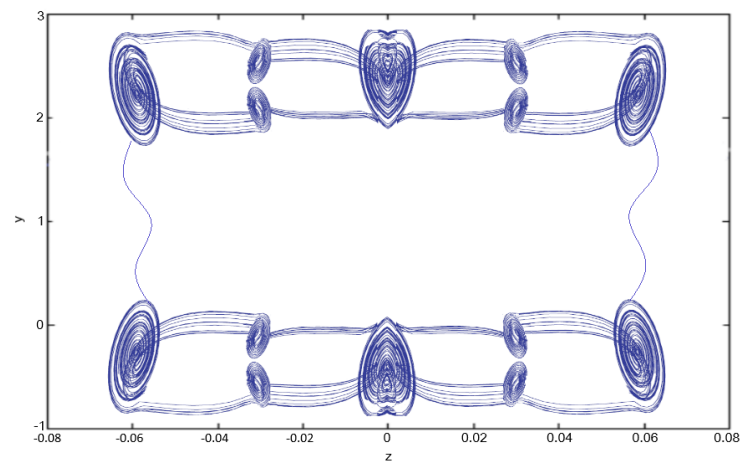

Figure 14. Self-similarity dynamic for the chaotic attractor with seven scrolls generated by the model (5.2)-(4.4) using the using the Legendre wavelets scheme with the parameter values $(b, d, W(\eta))=\left(\frac{1}{10}, \frac{1}{2}, \frac{1}{2} v\left(\eta-\frac{1}{2}\right)+\frac{1}{2} v\left(\eta-\frac{3}{2}\right)\right)$ and $\sigma=1$. We can see a set of two similar 7 -scroll chaotic attractors grouped to form a double symmetry around the axis $z=0$ in one side and $y=1$ in the other side.

It depicts the self-similarity process for the chaotic attractor with seven scrolls generated by the model (5.2)-(4.4) using the using the Legendre wavelets scheme with the parameter values $(b, d, W(\eta))=\left(\frac{1}{10}, \frac{1}{2}, \frac{1}{2} v\left(\eta-\frac{1}{2}\right)+\frac{1}{2} v\left(\eta-\frac{3}{2}\right)\right)$ and $\sigma=1$. It shows a set of two similar 7-scroll chaotic attractors grouped to form a double symmetry around the axis $z=0$ in one side and $y=1$ in the other side. 


\section{Conclusions}

Self-similarity processes are not only very important in image compressing and machining but also in network traffic prediction that represents both a huge academic problem and a concern for the industry and network performance environment. Moreover, some of other self-similarity's applications in real life consist of predicting number of biological dynamics or phenomena including bacteria growth pattern or nerve dendrite pattern. Hence, looking for alternative ways to reproduce and control self-similarity dynamics in a science laboratory, workstation or path lab has also become fascinating for researchers, especially since the phenomenon occurs naturally around us. Hence, we have used three major combined concepts, namely the step series switching process, the Julia's technique and fractal-fractional dynamic to generate various forms of self-similarity dynamics related to chaotic attractors which, initially comprise two, five and seven scrolls. The model obtained in each case has been solved using numerical techniques and related numerical simulations have been performed. We have noticed that the initial attractors can engage in the selfsimilarity dynamics, thereby generating the exact or approximately exact copy of themselves or part of themselves. Moreover, the dynamics of the copies have been proved to be influenced and controlled by some of the model's parameters involved in the process. This work improves the preceding ones with the application of three combined operations to generate self-similarity processes while maintaining some important properties such as symmetry, invariance of the number of scrolls, dynamical sense and orientation. This may then open more doors for engineers, mathematicians and computer scientists looking for deeper investigations in physical and mathematical concepts capable of re-generating features that already exist naturally in our environment.

\section{References}

[1] M.A. Alqudah, C. Ravichandran, T. Abdeljawad and N. Valliammal. New results on Caputo fractional-order neutral differential inclusions without compactness. Advances in Difference Equations, 528(2019):1-14, 2019. https://doi.org/10.1186/s13662-019-2455-z.

[2] A. Atangana and E.F.D. Goufo. Cauchy problems with fractal-fractional operators and applications to groundwater dynamics. Fractals, 28(08):2040043, 2020. https://doi.org/10.1142/S0218348X20400435.

[3] Abdon Atangana. Fractal-fractional differentiation and integration: Connecting fractal calculus and fractional calculus to predict complex system. Chaos, Solitons \& Fractals, 102:396-406, 2017. https://doi.org/10.1016/j.chaos.2017.04.027.

[4] K. Bouallegue. Gallery of chaotic attractors generated by fractal network. International Journal of Bifurcation and Chaos, 25(01):1530002, 2015. https://doi.org/10.1142/S0218127415300025.

[5] Y. Chen, X. Ke and Y. Wei. Numerical algorithm to solve system of nonlinear fractional differential equations based on wavelets method and the er- 
ror analysis. Applied Mathematics and Computation, 251:475-488, 2015. https://doi.org/10.1016/j.amc.2014.11.079.

[6] E.F. Doungmo Goufo. Solvability of chaotic fractional systems with 3D four-scroll attractors. Chaos, Solitons \& Fractals, 104:443-451, 2017. https://doi.org/10.1016/j.chaos.2017.08.038.

[7] E.F. Doungmo Goufo. On chaotic models with hidden attractors in fractional calculus above power law. Chaos, Solitons \& Fractals, 127:24-30, 2019. https://doi.org/10.1016/j.chaos.2019.06.025.

[8] E.F. Doungmo Goufo. Fractal and fractional dynamics for a 3D autonomous and two-wing smooth chaotic system. Alexandria Engineering Journal, 59(4):24692476, 2020. https://doi.org/10.1016/j.aej.2020.03.011.

[9] E.F. Doungmo Goufo. The Proto-Lorenz system in its chaotic fractional and fractal structure. International Journal of Bifurcation and Chaos, 30(12):2050180, 2020. https://doi.org/10.1142/S0218127420501801.

[10] E.F.D. Goufo and J.J. Nieto. Attractors for fractional differential problems of transition to turbulent flows. Journal of Computational and Applied Mathematics, 339:329-342, 2018. https://doi.org/10.1016/j.cam.2017.08.026.

[11] S. Hotton and J. Yoshimi. Extending dynamical systems theory to model embodied cognition. Cognitive Science, 35(3):444-479, 2011. https://doi.org/10.1111/j.1551-6709.2010.01151.x.

[12] Y. Khan. Maclaurin series method for fractal differential-difference models arising in coupled nonlinear optical waveguides. Fractals, 29(01):2150004, 2021. https://doi.org/10.1142/S0218348X21500043.

[13] Y. Khan, M. Fardi, K. Sayevand and M. Ghasemi. Solution of nonlinear fractional differential equations using an efficient approach. Neural Computing and Applications, 24(1):187-192, 2014. https://doi.org/10.1007/s00521-012-1208-7.

[14] Q. Li, X.-S. Yang and F. Yang. Multiple-scrolls chaotic attractor and circuit implementation. Electronics Letters, 39(18):1306-1307, 2003. https://doi.org/10.1049/el:20030847.

[15] J. Lu, X. Yu and G. Chen. Generating chaotic attractors with multiple merged basins of attraction: A switching piecewise-linear control approach. IEEE Transactions on Circuits and Systems I: Fundamental Theory and Applications, 50(2):198-207, 2003. https://doi.org/10.1109/TCSI.2002.808241.

[16] P. Melby, N. Weber and A. Hübler. Dynamics of self-adjusting systems with noise. Chaos: An Interdisciplinary Journal of Nonlinear Science, 15(3):033902, 2005. https://doi.org/10.1063/1.1953147.

[17] S.S. Motsa, Y. Khan and S. Shateyi. Application of piecewise successive linearization method for the solutions of the Chen chaotic system. Journal of Applied Mathematics, 2012, 2012. https://doi.org/10.1155/2012/258948.

[18] C. Ravichandran, K. Jothimani, H.M. Baskonus and N. Valliammal. New results on nondensely characterized integro differential equations with fractional order. The European Physical Journal Plus, 133(3):1-9, 2018. https://doi.org/10.1140/epjp/i2018-11966-3.

[19] C. Ravichandran, K. Logeswari, S.K. Panda and K.S. Nisar. On new approach of fractional derivative by Mittag-Leffler kernel to neutral integro-differential systems with impulsive conditions. Chaos, Solitons \& Fractals, 139:110012, 2020. https://doi.org/10.1016/j.chaos.2020.110012. 
[20] M. Razzaghi and S. Yousefi. The Legendre wavelets operational matrix of integration. International Journal of Systems Science, 32(4):495-502, 2001. https://doi.org/10.1080/00207720120227. 